\title{
Quantum Transport of Electrons Through a Parallel-Coupled Triple Quantum-Dot Molecule
}

\author{
M. L. Ladrón de Guevara and P. A. Orellana \\ Departamento de Física, Universidad Católica del Norte, Angamos 0610, Antofagasta, Chile
}

\section{Received on 8 December, 2005}

\begin{abstract}
In this article we study the electronic transport through a triple quantum-dot molecule parallel-coupled to leads under a magnetic field. Analytical expressions are obtained for both the conductance and total density of states for the molecule in equilibrium at zero temperature. As a result of quantum interference of resonances belonging to different channels, this configuration exhibits bound states in the continuum (BICs). We examine the broadenings of the molecular states around the conditions under which BICs occur, finding long-lived states extremely robust under variations of the magnetic flux.
\end{abstract}

Keywords: Electronic transport; Quantum dots; Quantum interference

\section{INTRODUCTION}

A distinctive feature of electron tunneling through quantum dots is the retention of the quantum phase coherence. For this reason, multiple connected geometries involving quantum dots exhibit quantum interference effects. Several works have been concerned with the study of transmission through a double quantum dot molecule embedded in an AharonovBohm interferometer[1-5]. This system is characterized by the formation of a tunable Fano resonance in the conductance spectrum. This resonance is associated to a long-lived molecular state, where the lifetime is controlled by the magnetic field. For specific values of the magnetic flux, this molecular state becomes totally uncoupled from the leads[3, 6], and a "bound state in the continuum" (BIC) is formed. This state immersed in a continuum is the result of the interference of resonances belonging to different channels. These states were early predicted by von Neumann and Wigner[7]. Much later, BICs were studied in the context of atomic and molecular physics[8] and more recently in mesoscopic systems[9].

In this article we study the electronic transport through a parallel triple quantum-dot molecule embedded in an Aharonov-Bohm interferometer connected symmetrically to leads. We focus our analysis in a particular set of values of the dot-lead couplings, where two BICs can occur simultaneously. We obtain analytical expressions for the conductance and total density of states, and we explore them as a function of the magnetic flux to demonstrate that different regimes of transmission can be reached. The conductance displays one Breit-Wigner and two Fano resonances at the energies of the molecular states, and with a period of two flux quanta, the roles of the antibonding and bonding states are interchanged in the transmission spectrum. The simultaneous BICs occur when the magnetic flux is a multiple of $2 \Phi_{0}$, but in a wide range around this value the pair of states keeps very weakly coupled to the leads. Approximate expressions of the line broadenings of the molecular states are given, as functions of the Aharonov-Bohm phase.
II. MODEL

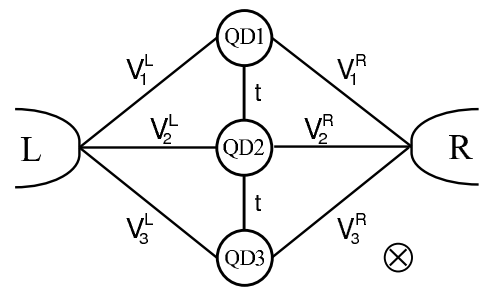

FIG. 1: Triple quantum dot molecule coupled in parallel to leads.

We consider three single-level quantum dots forming a triple quantum dot molecule coupled in parallel to two leads, as shown in Fig.1. The system is modeled by a noninteracting Anderson Hamiltonian, which can be written as

$$
H=H_{m}+H_{l}+H_{I},
$$

where $H_{m}$ describes the dynamics of the isolate molecule,

$$
H_{m}=\sum_{i=1}^{3} \varepsilon_{i} d_{i}^{\dagger} d_{i}-t\left(d_{1}^{\dagger} d_{2}+d_{2}^{\dagger} d_{1}\right)-t\left(d_{2}^{\dagger} d_{3}+d_{3}^{\dagger} d_{2}\right),
$$

where $\varepsilon_{i}$ is the level energy of dot $i, d_{i}\left(d_{i}^{\dagger}\right)$ annihilates (creates) an electron in dot $i$, and $t$ is the interdot tunneling coupling. $H_{l}$ is the Hamiltonian for the noninteracting electrons in the left and right leads

$$
H_{l}=\sum_{k \in L, R} \varepsilon_{k} c_{k}^{\dagger} c_{k}
$$

where $c_{k}\left(c_{k}^{\dagger}\right)$ is the annihilation (creation) operator of an electron of quantum number $k$ and energy $\varepsilon_{k}$ in the contact $L$ or $R$. The term $H_{I}$ accounts for the tunneling between dots and leads,

$$
H_{I}=\sum_{i=1}^{3} \sum_{k \in L} V_{i}^{L} d_{i}^{\dagger} c_{k}+\text { h. c. }+\sum_{i=1}^{3} \sum_{k \in R} V_{i}^{R} d_{i}^{\dagger} c_{k}+\text { h. c. }
$$


with $V_{i}^{L(R)}$ the tunneling matrix element connecting the $i-$ th dot with the left (right) lead, assumed independent of $k$. For simplicity, we assume that the magnitudes of these matrix elements are such as $\left|V_{1}^{L}\right|=\left|V_{1}^{R}\right| \equiv V_{1},\left|V_{2}^{L}\right|=\left|V_{2}^{R}\right| \equiv V_{2}$, and $\left|V_{3}^{L}\right|=\left|V_{3}^{R}\right| \equiv V_{3}$. In presence of a magnetic field, and in the symmetric gauge, the tunnel matrix elements can be written in the form

$$
\begin{aligned}
& V_{1}^{L}=V_{1} e^{-i \phi / 4}, \quad V_{1}^{R}=V_{1} e^{i \phi / 4}, \\
& V_{2}^{L}=V_{2}^{R}=V_{2}, \\
& V_{3}^{L}=V_{3} e^{i \phi / 4}, \quad V_{3}^{R}=V_{3} e^{-i \phi / 4},
\end{aligned}
$$

with $\phi=2 \pi \Phi / \Phi_{0}$, the Aharonov-Bohm phase, where $\Phi_{0}=$ $h / e$ is the flux quantum.

The linear conductance at zero temperature is given by the Landauer formula

$$
G=\frac{2 e^{2}}{h} T\left(\varepsilon_{F}\right),
$$

where $T(\varepsilon)$ is the total transmission. To obtain $G$ explicitly we use the equation of motion approach for the Green's functions[10]. The transmission can be expressed in terms of the retarded and advanced Green's functions $\mathbf{G}^{r / a}(\varepsilon)$ as

$$
T(\varepsilon)=\operatorname{tr}\left\{\mathbf{G}^{a}(\varepsilon) \Gamma^{R} \mathbf{G}^{r}(\varepsilon) \Gamma^{L}\right\},
$$

where $\mathbf{G}^{r}(\varepsilon)$ is defined by

$$
G_{i j}^{r}(t)=-i \theta(t)\left\langle\left\{d_{i}(t), d_{j}^{\dagger}(0)\right\}\right\rangle, \quad i, j=1,2,3,
$$

with $\theta(t)$ the step function. $\mathbf{G}^{a}(\varepsilon)$ is given by $G_{i j}^{a}=\left[G_{j i}^{r}\right]^{*}$, and $\Gamma^{L, R}$ are matrices describing the coupling between the quantum dots and the left and right leads, the matrix elements of which are

$$
\Gamma_{i j}^{L(R)}=2 \pi \sum_{k} V_{i k}^{L(R)}\left[V_{j k}^{L(R)}\right]^{*} \delta\left(\varepsilon-\varepsilon_{k}\right), \quad i, j=1,2,3 .
$$

With the use of Eq. (5), $\Gamma^{L, R}$ can be written as

$$
\Gamma^{L, R}=\left(\begin{array}{ccc}
\gamma_{11} & e^{\mp i \phi / 4} \gamma_{12} & e^{\mp i \phi / 2} \gamma_{13} \\
e^{ \pm i \phi / 4} \gamma_{21} & \gamma_{22} & e^{\mp i \phi / 4} \gamma_{23} \\
e^{ \pm i \phi / 2} \gamma_{31} & e^{ \pm i \phi / 4} \gamma_{32} & \gamma_{33}
\end{array}\right)
$$

with $\gamma_{i j} \equiv \Gamma_{i j}^{L}=\Gamma_{i j}^{R}$, where $\Gamma_{i j}^{L, R}$ are obtained from (9) for zero magnetic flux.

The electronic properties of the configuration can be studied from the total density of states, given by

$$
\rho(\varepsilon)=\sum_{i=1}^{3} \rho_{i}(\varepsilon)=-\frac{1}{\pi} \sum_{i=1}^{3} \operatorname{I} m G_{i i}^{r}(\varepsilon), \quad i=1,2,3,
$$

where $G^{r}(\varepsilon)$ is the retarded Green's function.

Hereafter we assume $\varepsilon_{1}=\varepsilon_{2}=\varepsilon_{3} \equiv \varepsilon_{0}$. We make the following transformation of the quantum-dot operators

$$
\begin{aligned}
& \bar{d}_{1}=\frac{1}{2}\left(d_{1}+\sqrt{2} d_{2}+d_{3}\right), \\
& \bar{d}_{2}=\frac{1}{\sqrt{2}}\left(d_{1}-d_{3}\right), \\
& \bar{d}_{3}=\frac{1}{2}\left(d_{1}-\sqrt{2} d_{2}+d_{3}\right),
\end{aligned}
$$

so that the Hamiltonian of the isolated molecule becomes diagonal

$$
\bar{H}_{m}=\left(\varepsilon_{0}+\sqrt{2} t\right) \bar{d}_{1}^{\dagger} \bar{d}_{1}+\varepsilon_{0} \bar{d}_{2}^{\dagger} \bar{d}_{2}+\left(\varepsilon_{0}-\sqrt{2} t\right) \bar{d}_{3}^{\dagger} \bar{d}_{3},
$$

and the Hamiltonian describing the coupling between the molecule and the leads takes the form

$$
\bar{H}_{I}=\sum_{i=1}^{3} \sum_{k \in L} \bar{V}_{i}^{L} \bar{d}_{i}^{\dagger} c_{k}+\text { h. c. }+\sum_{i=1}^{3} \sum_{k \in R} \bar{V}_{i}^{R} \bar{d}_{i}^{\dagger} c_{k}+\text { h. c. }
$$

where

$$
\begin{aligned}
& \bar{V}_{1}^{L, R}=\frac{1}{2}\left(V_{1}^{L, R}+\sqrt{2} V_{2}^{L, R}+V_{3}^{L, R}\right), \\
& \bar{V}_{2}^{L, R}=\frac{1}{\sqrt{2}}\left(V_{1}^{L, R}-V_{3}^{L, R}\right), \\
& \bar{V}_{3}^{L, R}=\frac{1}{2}\left(V_{1}^{L, R}-\sqrt{2} V_{2}^{L, R}+V_{3}^{L, R}\right) .
\end{aligned}
$$

Eqs. (5)-(15) give us interesting insight into the transmission properties of the molecule. It is observed that for some specific values of the magnetic flux and the dot-lead matrix elements, the coupling between one or more molecular states with the leads may vanish, giving rise to the formation of a BIC. In particular, if $V_{1}=V_{3}$,

$$
\begin{aligned}
& \bar{V}_{1}^{L, R}=2 V_{1} \cos \frac{\phi}{4}+\sqrt{2} V_{2}, \\
& \bar{V}_{2}^{L, R}=\mp i \sqrt{2} V_{1} \sin \frac{\phi}{4}, \\
& \bar{V}_{3}^{L, R}=2 V_{1} \cos \frac{\phi}{4}-\sqrt{2} V_{2} .
\end{aligned}
$$

So that when $\phi=4 n \pi$ ( $n$ integer), the matrix elements between the molecular state 2 and the left and right leads, $\bar{V}_{2}^{L, R}$, cancel and such a state becomes a BIC. If it also occurs that $V_{1}=V_{2}$ and $n$ is an odd multiple of $\pi, \bar{V}_{2}^{L, R} \neq 0$ but either $\bar{V}_{1}^{L, R}$ or $\bar{V}_{3}^{L, R}$ vanish, occurring again a bound state in the continuum. On the other hand, we can see of Eq. (16) that if $V_{1}=V_{3}$ and $V_{2}=\sqrt{2} V_{1}$, two BIC's are simultaneously formed when $\phi=$ $4 n \pi$ : one in the state $2\left(\varepsilon=\varepsilon_{0}\right)$, and other either in state 1 ( $\varepsilon=$ $\left.\varepsilon_{0}-\sqrt{2} t\right)$ or $3\left(\varepsilon=\varepsilon_{0}+\sqrt{2} t\right)$, depending on the parity of $n$. In a parallel double quantum dot molecule, a seemingly simpler condition gives rise to one BIC, which is formed whenever $\phi$ is a even multiple of $\pi$ (that is, $\Phi=n \Phi_{0}, n$ integer) [3, 6]. Notice that BICs occur for an infinite number of combinations of dot-lead couplings and Aharonov-Bohm phases, but for simplicity in what follows we focus in the case in that $V_{1}=$ $V_{3} \equiv V$ and $V_{2}=\sqrt{2} V$.

\section{CONDUCTANCE AND DENSITY OF STATES}

When $V_{1}=V_{3}=V$ and $V_{2}=\sqrt{2} V$ the linear conductance reduces to

$$
G=\frac{2 e^{2}}{h} \frac{4 \gamma^{2}\left[\left(t+\sqrt{2} \varepsilon \cos \frac{\phi}{4}\right)^{2}-t^{2} \cos \frac{\phi}{2}\right]^{2}}{D_{1}(\varepsilon) D_{2}(\varepsilon)} .
$$


where

$$
D_{1}(\varepsilon)=\varepsilon^{2}+\gamma^{2}\left(1-\cos \frac{\phi}{2}\right)^{2}
$$

and

$$
D_{2}(\varepsilon)=\left(2 t^{2}-\varepsilon^{2}\right)^{2}+\gamma^{2}\left[4 \sqrt{2} t \cos \frac{\phi}{4}+\varepsilon\left(3+\cos \frac{\phi}{2}\right)\right]^{2}
$$

Figure 2 shows the conductance spectrum for different values of the Aharonov-Bohm phase. For $\phi=0$ (Fig.2(a)) the conductance exhibits a single resonance around the antibonding energy, which indicates that transmission through the other two molecular states has been suppressed, and such states are bound states in the continuum. This is consistent with Ecs. (16), which show that both $\bar{V}_{2}^{L, R}$ and $\bar{V}_{3}^{L, R}$ cancel whenever $\phi=8 n \pi$ ( $n$ integer). The equations show also that the roles of the bonding and antibonding states are inverted every $4 \pi$, and that the antibonding and the molecular state of intermediate energy both collapse to BIC's when $\phi=4 \pi(2 n-1)$ ( $n$ integer).

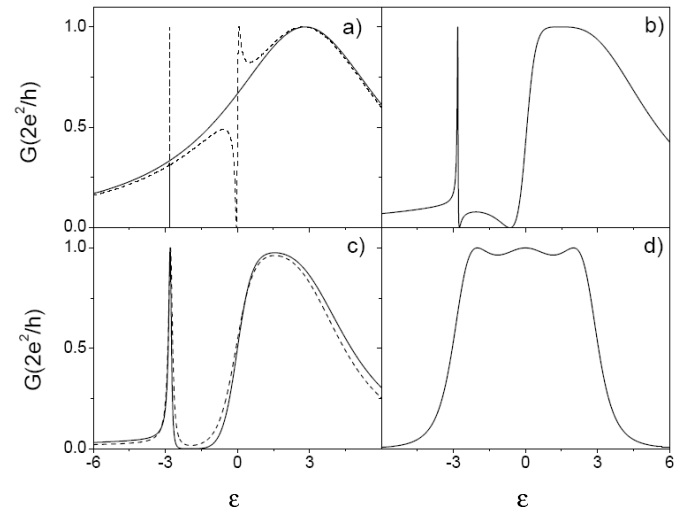

FIG. 2: Dimensionless conductance versus Fermi energy, in units of $\gamma$, for $V_{1}=V_{3}=V, V_{2}=\sqrt{2} V, t=2$, a) $\phi=0$ (solid line) and $\phi=\pi / 5$ (dash line), b) $\phi=3 \pi / 4$, c) $\phi=\pi$ (solid line) and $\phi=6 \pi / 5$ (dash line), d) $\phi=2 \pi$, and $\varepsilon_{0}=0$.

For arbitrary values of the Aharonov-Bohm phase the spectrum presents three resonances, and a number of Fano antiresonances that oscillates between two and zero. From Eq. (17) we note that the conductance is zero at

$$
\varepsilon=\frac{-\sqrt{2} t[1 \pm \sqrt{\cos (\phi / 2)}]}{2 \cos \phi / 4},
$$

where we see that when $(4 n-1) \pi<\phi<(4 n+1) \pi$ ( $n$ integer) there are two antiresonaces, as shown by Fig. 2(b) where $\phi=$ $3 \pi / 4$. When $\phi=n \pi$ ( $n$ odd) only one point of zero conductance exists, as observed in (c). For $(4 n-3) \pi<\phi<(4 n-1) \pi$ ( $n$ integer) the numerator of Eq. (17) is complex and the conductance does not exhibit antiresonances (Figs. (c), dashed line, and (d)). In (d) $G(\varepsilon)$ is symmetrical around $\varepsilon=0$, and has the form

$$
G=\frac{2 e^{2}}{h} \frac{16 \gamma^{2} t^{4}}{\left(4 \gamma^{2}+\varepsilon^{2}\right)\left(4 t^{4}-4(t+\gamma)(t-\gamma) \varepsilon^{2}+\varepsilon^{4}\right)},
$$

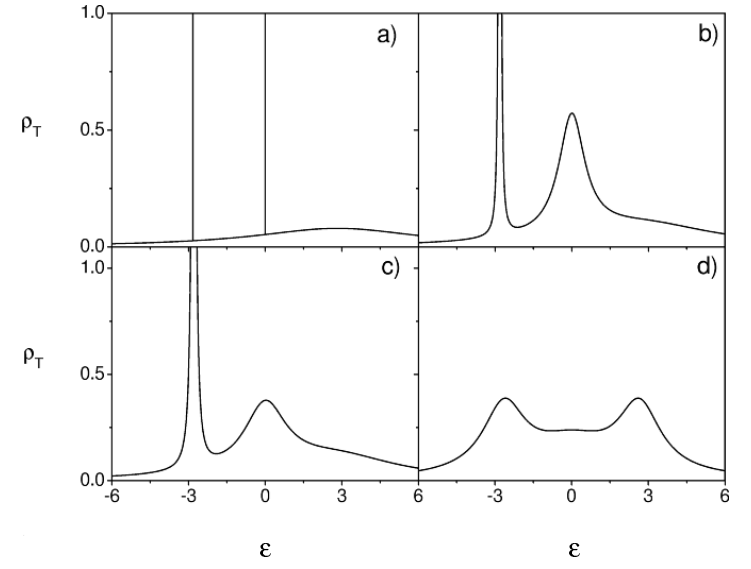

FIG. 3: Density of state versus Fermi energy, in units of $\gamma$, for $V_{1}=$ $V_{3}=V, V_{2}=\sqrt{2} V, t=2$, a) $\phi=0$, b) $\phi=3 \pi / 4$, c) $\phi=\pi$ and d) $\phi=2 \pi$, for $\varepsilon_{0}=0$

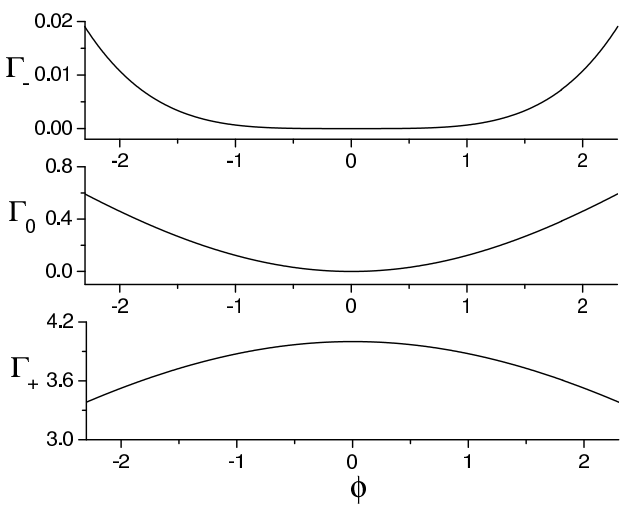

FIG. 4: Broadenings of the molecular states $\Gamma_{-}, \Gamma_{0}$ and $\Gamma_{+}$as a function of $\phi$, for $V_{1}=V_{3}=V, V_{2}=\sqrt{2} V$ and $t=2$.

which corresponds exactly to the conductance of a triple quantum dot molecule connected in series. It is interesting to note that if the dots are not coupled directly, that is, $t=0$, the transmission is suppressed for all energies (perfect reflector). An analogous result is found in two parallel quantum dots in presence of a magnetic field. This situation never occurs in the triple molecule when the dot-lead couplings are equal.

The total density of states is given by

$$
\begin{array}{r}
\rho_{T}=\frac{\gamma}{\pi} \frac{\left[8 \sqrt{2} t \varepsilon \cos \frac{\phi}{4}+\left(2 t^{2}+\varepsilon^{2}\right)\left(3+\cos \frac{\phi}{2}\right)\right]}{\left(2 t^{2}-\varepsilon^{2}\right)^{2}+\gamma^{2}\left[4 \sqrt{2} t \cos \frac{\phi}{4}+\varepsilon\left(3+\cos \frac{\phi}{2}\right)\right]^{2}} \\
+\frac{\gamma}{\pi} \frac{2 \sin ^{2} \frac{\phi}{4}}{\varepsilon^{2}+4 \gamma^{2} \sin ^{4} \frac{\phi}{4}} .
\end{array}
$$

In the range $2(4 n-3 / 8) \pi \leq \phi \leq 2(4 n+3 / 8) \pi$ ( $n$ integer), $\rho_{T}$ 
can be approximated by a sum of Lorentzians of the form

$$
\begin{array}{r}
\rho_{T} \approx \frac{C}{\pi}\left[\frac{\Gamma_{-}}{\Gamma_{-}^{2}+\left(\varepsilon-\varepsilon_{-}\right)^{2}}+\frac{\Gamma_{+}}{\Gamma_{+}^{2}+(\varepsilon-\sqrt{2} t)^{2}}\right]+ \\
+\frac{1}{\pi} \frac{\Gamma_{0}}{\Gamma_{0}^{2}+\varepsilon^{2}},
\end{array}
$$

where

$$
\begin{gathered}
\varepsilon_{-}=\frac{-4 \sqrt{2} t\left(2 t^{2}+\gamma^{2} \cos (\phi / 4)[3+\cos (\phi / 2)]\right)}{\left(8 t^{2}+\gamma^{2}[3+\cos (\phi / 2)]^{2}\right)}, \\
C=\frac{4 \cos ^{4} \frac{\phi}{4}}{3+\cos \frac{\phi}{2}}
\end{gathered}
$$

and the broadenings are given by

$$
\begin{gathered}
\Gamma_{-}=8 \gamma \frac{t^{2} \sin ^{4} \frac{\phi}{8}}{2 t^{2}+\gamma^{2}\left(1+\cos ^{2} \frac{\phi}{4}\right)^{2}} \\
\Gamma_{0}=2 \gamma \sin ^{2} \frac{\phi}{4} \quad \text { and } \quad \Gamma_{+}=4 \gamma \cos ^{4} \frac{\phi}{8}
\end{gathered}
$$

Figure 3 shows $\rho_{T}$ for the same parameters of Fig.2. In figure Fig.3(a), where $\phi=0$, the density of states is the superposition of two Dirac delta's localized at $\varepsilon=\varepsilon_{-}$and $\varepsilon=0$ (corresponding to the BIC's) plus a Lorentzian at $\varepsilon=\varepsilon_{+}$with width $4 \gamma$. When $\phi=2 \pi$, as in (d), the density of states correspond to that of a triple molecule connected in series.
Figure 4 displays the broadenings $\Gamma_{-}, \Gamma_{0}$ and $\Gamma_{+}$as a function of $\phi$, for $t=2$, in the range of validity of Eqs. (24).

The top plot shows the BIC formed at the bonding state when $\phi=0$ and the robustness of such a long-lived state against variations of the magnetic field. Notice that $\Gamma_{-}$remains smaller than 0.02 in all the range of $\phi$, and that is very close to zero in a wide interval around $\phi=0$. For instance, for $\phi \in(-\pi / 3, \pi / 3), \Gamma_{-}$keeps smaller than $8 \times 10^{-4}$, that is, less than 0.08 per cent of the level broadening of a single quantum dot. The broadening $\Gamma_{0}$ is more sensitive to variations of the magnetic field than $\Gamma_{-}$, as shown in the middle figure.

\section{CONCLUSIONS}

We have investigated the electron transport through a parallel-coupled triple quantum dot molecule in presence of a magnetic field. For the studied case, where $V_{1}=V_{3}=V$ and $V_{2}=\sqrt{2} V$, different regimes of transmission are possible as the magnetic flux is varied; if this is a multiple of $2 \Phi_{0}$ two BICs simultaneous are formed, and in a wide range around this value the two resonances keep extremely narrow.

\section{Acknowledgments}

The authors would like to thank financial support from FONDECYT under grants 1040385 and 1020269. M. L. L. de G. receives financial support from Milenio ICM P02-049$\mathrm{F}$ and P. A. O. from Milenio ICM P02-054-F.
[1] Kicheon Kang and Sam Young Cho, J. Phys: Condens. Matter 16, 117 (2004)

[2] Zhi-Ming Bai, Min-Fong Yang, and Yung-Chung Chen, J. Phys.: Condens. Matter 16, 2053 (2004).

[3] Pedro A. Orellana, M. L. Ladrón de Guevara, and F. Claro Phys. Rev. B 70, 233315 (2004).

[4] Guo-Hui Ding, Chul Koo Kim, and Kyun Nahm, Phys. Rev. B, 71205313 (2005).

[5] Haizhou Lu, Rong Lü, and Bang-fen Zhu, Phys. Rev. B 71 , 235320 (2005).

[6] M. L. Ladrón de Guevara, F. Claro, and Pedro A. Orellana, Phys. Rev. B 67, 195335 (2003).

[7] J. von Neumann and E. Wigner, Phys. Z. 30, 465 (1929); Frank H. Stillinger and David R. Herrick, Phys. Rev. A 11, 446
(1975).

[8] H. Friedrich and D. Wintgen, Phys. Rev. A 32, 3231 (1985); S. L. Haan and G. S. Agarwal, Phys. Rev. A 35, 4592 (1987); P. R. Levstein, H. M. Pastawskit, J. L. D’Amato, J. Phys.: Condens. Matter, 2, 1781 (1990).

[9] J. U. Nöckel, Phys. Rev. B 4615348 (1992); O. Olendski and L. Mikhailovska, Phys. Rev B 67, 035310 (2003); F. Guinea and J. A. Vergés Phys. Rev. B 35, 979 (1987); J. L. D’Amato, H. M. Pastawski, and J. F. Weisz, Phys. Rev. B 39, 3554 (1989); I. Rotter and F. Sadreev, Phys. Rev. E 71, 046204 (2005).

[10] Supriyo Datta, "Electronic transport in mesoscopic systems" (Cambridge Univ. Press, 1997). 